\title{
Two-Element Phased Array of Anti-Guided Vertical-Cavity Lasers
}

Darwin K. Serkland, K: D. Choquette, G. R. Hadley, K. M. Geib, and A. A. Allerman

Sandia National Laboratories, P.O. Box 5800, MS 0603, Albuquerque, NM 87185

\begin{abstract}
:
We demonstrate for the first time anti-guided coupling of two adjacent vertical-cavity surface-emitting lasers (VCSEL's), obtaining a 1-by-2 phase-locked array at $869 \mathrm{~nm}$. The lateral index modification required for anti-guiding is achieved by a patterned 3-nm etch performed between two epitaxial growths. In contrast with prior evanescently coupled VCSEL's, adjacent anti-guided VCSEL's can emit in-phase and produce a single on-axis lobe in the far field. Greater than $2 \mathrm{~mW}$ of in-phase output power is demonstrated with two VCSEL's separated by $8 \mu \mathrm{m}$. Moreover, phase locking of two VCSEL's separated by $20 \mu \mathrm{m}$ is observed, indicating the possibility of a new class of optical circuits based upon VCSEL's that interact horizontally and emit vertically.
\end{abstract}

Index Terms: diode laser arrays, phased arrays, vertical cavity surface emitting lasers, optical resonators, semiconductor lasers.

\author{
Corresponding Author: \\ Darwin K. Serkland \\ Tel. (505) 844-5355 \\ Fax (505) 844-8985 \\ Email: dkserk1@sandia.gov
}

(Submitted to Applied Physics Letters) 


\section{DISCLAIMER}

This report was prepared as an account of work sponsored by an agency of the United States Government. Neither the United States Government nor any agency thereof, nor any of their employees, make any warranty, express or implied, or assumes any legal liability or responsibility for the accuracy, completeness, or usefulness of any information, apparatus, product, or process disclosed, or represents that its use would not infringe privately owned rights. Reference herein to any specific commercial product, process, or service by trade name, trademark, manufacturer, or otherwise does not necessarily constitute or imply its endorsement, recommendation, or favoring by the United States Government or any agency thereof. The views and opinions of authors expressed herein do not necessarily state or reflect those of the United States Government or any agency thereof. 


\section{DISCLAIMER}

Portions of this document may be illegible in electronic image products. Images are produced from the best available original document. 
The high efficiency, low threshold current, and wafer-scale fabrication of vertical-cavity surfaceemitting lasers (VCSEL's) make them attractive for many applications. However, for applications that require more than $5 \mathrm{~mW}$ of power in a diffraction-limited beam, standard VCSEL's are unsuitable. Although the output power scales with the area, typical VCSEL's with diameters larger than $5 \mu \mathrm{m}$ emit into many transverse modes and are not diffraction limited. Phase-locked arrays of VCSEL's offer a means of achieving higher-power diffraction-limited beams. Previous works have reported phase-locked VCSEL arrays that tended to emit light into the highest order transverse mode, ${ }^{1,2,3}$ as is characteristic of evanescently coupled lasers. In this Letter, we demonstrate for the first time phase locking of two VCSEL's by means of anti-guided coupling. Anti-guided coupling permits in-phase emission and phase locking of widely separated lasers.

The two principal techniques for phase locking semiconductor lasers are evanescent-wave coupling and anti-guided coupling. Evanescent-wave coupling has several limitations: the coupling per unit length is weak, adjacent elements tend to emit out of phase, and there is very little modal selectivity between the various array super-modes. In order to overcome these limitations in edge-emitting laser arrays, the technique of anti-guided coupling was introduced, where the refractive index was increased in between the active gain regions. ${ }^{4,5}$ Light emitted in one of the active array elements is not confined by total internal reflection, but rather can easily "leak" to adjacent array elements. It has been shown that antiguided coupling provides stronger coupling and increased modal discrimination by enhancing the loss of undesired array modes. ${ }^{6}$ Moreover, the array elements can emit in phase, if the index step and the width of the coupling region are properly chosen.

Hadley recently proposed a novel technique of tailoring the effective index by modifying the optical cavity length as indicated in Fig. 1(a). ${ }^{7}$ In contrast, previous techniques have required deposition of different materials side by side. ${ }^{8}$ Hadley theoretically showed that a shift in the longitudinal resonance wavelength yields a corresponding change of the effective index, where a blue shift of the cavity resonance decreases the effective index. For VCSEL's, the cavity length modification can be achieved by etching the cavity spacer layer through a laterally patterned mask before deposition of the top mirror layers. This technique was recently applied to make single-element anti-guided VCSEL's that remained 
in the fundamental transverse mode far above threshold. ${ }^{9,10}$ Figure 1(a) illustrates the scheme for two adjacent VCSEL's whose cavity lengths are decreased slightly as compared to the coupling region between them. Figure 1(b) shows a plot of the longitudinal k-vector component, determined by the longitudinal resonance condition, versus lateral position. Within each etched region, light is emitted normal to the surface, as indicated by the vertical vector $\mathbf{k}$ in Fig. 1(b). Light can easily "leak" out of the etched region and into the surrounding area, provided that the k-vector tilts, as shown by the vector $\mathbf{k}^{\prime}$ in Fig. 1(b), satisfying the longitudinal resonance condition outside of the etched region. The illustration suggests that a transverse standing wave exists in the coupling region between the two VCSEL elements, due to the presence of counter-propagating transverse k-vector components.

We have fabricated structures containing two anti-guided VCSEL's separated by 8 to $20 \mu \mathrm{m}$. The desired effective index modification was obtained by performing a patterned shallow etch between two epitaxial growths. Starting with an n-type GaAs substrate, we used metal-organic vapor-phase epitaxy (MOVPE) to grow the bottom n-type distributed Bragg reflector (DBR), the one-wavelength-long optical cavity, and three quarters of the first period of the top p-type DBR. The DBR periods consisted of high and low-index layers of $\mathrm{Al}_{0.16} \mathrm{Ga}_{0.84} \mathrm{As}$ and $\mathrm{Al}_{0.92} \mathrm{Ga}_{0.08} \mathrm{As}$, respectively. The $\mathrm{Al}_{0.92} \mathrm{Ga}_{0.08} \mathrm{As}$ layer is replaced by $\mathrm{Al}_{0.98} \mathrm{Ga}_{0.02} \mathrm{As}$ in the DBR periods immediately adjacent to the optical cavity in order to permit lateral oxidation for the realization of current apertures. ${ }^{11}$ The bottom DBR consists of 36 periods, including the period intended for oxidation. The optical cavity contains 5 GaAs quantum wells, each 8$\mathrm{nm}$ thick, surrounded by $8-\mathrm{nm}$ barriers of $\mathrm{Al}_{0.2} \mathrm{Ga}_{0.8} \mathrm{As}$. After the first growth, we etched $3 \mathrm{~nm}$ of the top $\mathrm{Al}_{0.16} \mathrm{Ga}_{0.84} \mathrm{As}$ layer through openings in a lithographically patterned photoresist mask. Oxides that might have formed at the reactive $\mathrm{Al}_{0.16} \mathrm{Ga}_{0.84}$ As surface were removed by rinsing the wafer with $\mathrm{NH}_{4} \mathrm{OH}$ and de-ionized water before transferring it back into the MOVPE growth chamber. The second MOVPE growth commenced with a p-type $\mathrm{Al}_{0.16} \mathrm{Ga}_{0.84} \mathrm{As}$ layer, completing the first DBR period, and continued with another 20 standard p-type DBR periods including the final GaAs cap layer.

The inset in Fig. 2 schematically shows a top view of the coupled VCSEL structure. The two inner squares (shaded gray) indicate where the 3-nm shallow etch was performed to lower the effective index within 4-by-4- $\mu \mathrm{m}$ regions. We note that the $3-\mathrm{nm}$ etch theoretically yields a $1.6-\mathrm{nm}$ decrease in the 
longitudinal resonance wavelength, which translates into a 0.006 decrease in the effective index. The $\mathrm{Al}_{0.98} \mathrm{Ga}_{0.02} \mathrm{As}$ layers were laterally oxidized (hatched region of the inset) to a distance of $14.5 \mu \mathrm{m}$ from the edges of the rectangular mesa, in order to confine the current and provide strong index confinement surrounding the coupled VCSEL's. The black regions of the inset represent the metal electrodes that contacted the top p-type GaAs cap layer. Due to the electrode arrangement and the bulk resistivity of the top DBR mirror, injecting current into the left (right) electrode yielded a high current density through the left (right) etched square. Injecting roughly equal currents into both the left and right electrodes resulted in phase-locked emission from the two VCSEL's.

Figure 2 shows the total output power from the two VCSEL's (separated by $8 \mu \mathrm{m}$ ) versus the total applied current. The data was measured with the currrent relationship $I_{L}=0.85 I_{R}$, where $I_{L}$ and $I_{R}$ denote the currents injected into the left and right electrodes, respectively. This slight current imbalance was required to maintain in-phase operation, presumably due to a slight asymmetry between the two VCSEL's. If the currents were significantly unbalanced, the output switched from the in-phase to the outof-phase mode, presumably due to asymmetric heat-induced index changes. Because of the long resonant wavelength $(869 \mathrm{~nm})$, resulting from a slightly fast growth rate, we typically heated the sample to $50{ }^{\circ} \mathrm{C}$ in order to align better the peak-gain wavelength with the cavity resonance. For total current levels above $10 \mathrm{~mA}$ in Fig. 2, the VCSEL's emitted in-phase as shown in Fig. 3(a). For currents between 6.5 and 9.5 $\mathrm{mA}$, the output appeared as a superposition of the in-phase and out-of-phase modes, with the out-of-phase mode emitting at a wavelength $0.7 \mathrm{~nm}$ shorter than the in-phase mode.

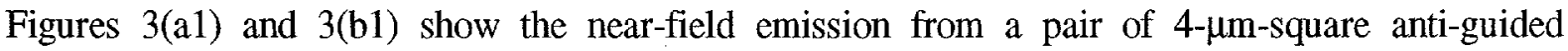
VCSEL's separated by 8 and $20-\mu \mathrm{m}$ coupling regions, respectively. Imaging into a spectrometer revealed that both VCSEL's emitted at the same wavelength (nominally $869 \mathrm{~nm}$ ). Moreover, phase locking was confirmed by the stable far-field interference patterns shown in the second row of Fig. 3 . The constructive interference along the axis between the two VCSEL's in Fig. 3(a2) indicates that the closely spaced elements emitted in-phase. In contrast, the destructive interference between the two VCSEL's in Fig. 3(b2) indicates that the widely spaced elements emitted out-of-phase, which is typical of prior evanescent-wave-coupled VCSEL arrays. Finally, the third row of Fig. 3 shows single-VCSEL far-field 
intensity profiles, obtained by driving current into one electrode only (the left one). The width of the central far-field lobe in Fig. 3(a2) indicates a narrowed angular divergence of the beam, determined by the distance between the two VCSEL's, as compared to the wider angular divergence of Fig. 3(a3), determined by the width of the fundamental mode of a single VCSEL.

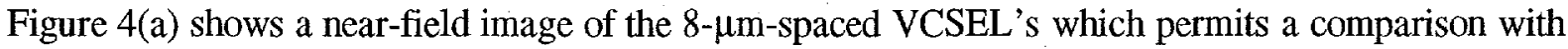
the numerical simulation in Fig. 4(b). The numerical calculations include the strong index confinement of the oxide layers at the left and right edges of the structure. As anticipated from the intuitive picture in Fig. 1(b), a transverse standing-wave pattern exists in the coupling region, where the number of intermediate intensity lobes scales with the product of the transverse k-vector and the length of the coupling region. For etch depths of 2.5 and $3.0 \mathrm{~nm}$, the two-dimensional simulations predict three lobes in the coupling region. In Fig. 4(a), we believe that the center electrode obscures the middle intermediate lobe that appears in the simulation in Fig. 4(b). As indicated by the plus and minus signs in Fig. 4(b), adjacent lobes are $180^{\circ}$ out of phase. Hence, an odd number of intermediate lobes, as shown in Fig. 4(b), corresponds to in-phase emission from the outer two lobes, as confirmed by the far-field image of Fig. 3(a2). The simulations required a $2.5-\mathrm{nm}$ etch depth (in agreement with the measured 3-nm depth) to match the observed out-of-phase operation of the 20- $\mu \mathrm{m}$ spaced VCSEL's and predicted 8 intermediate lobes.

Although we have demonstrated phase-locking of two anti-guided VCSEL's, further device improvements are desired. First, the shallow 3-nm-etch depth yielded a small resonance-wavelength shift and corresponding effective index step. Consequently, the phasing of the VCSEL's was susceptible to current-induced heating, which increased the index enough to produce wavelength shifts comparable to the 1.6-nm shift arising from the 3-nm etch. This likely explains why large current imbalances caused switching between in-phase and out-of-phase operation. A somewhat deeper etch should produce devices that are less sensitive to current imbalances. Next, the strong index confinement arising from the lateral oxidation is expected to reduce the modal selectivity of phased arrays. We are currently investigating structures with less index confinement, achieved by using proton-implantation to define the current 
aperture. The higher-order array modes are expected to suffer greater radiation losses at the perimeter of the implanted arrays and therefore be suppressed.

In conclusion, we have demonstrated phase-locking of two adjacent VCSEL's by means of antiguided coupling. Both in-phase and out-of-phase operation were obtained, in agreement with twodimensional numerical simulations. The observed phase locking of two VCSEL's separated by $20 \mu \mathrm{m}$ is particularly noteworthy since it would have been impossible to achieve by evanescent-wave coupling. This result suggests the possibility of a new type of optical integrated circuit consisting of VCSEL's that interact horizontally and emit vertically. Additionally, beam steering and switching devices based on anti-guided VCSEL's could be realized by tuning the effective index step and hence the transverse component of the k-vector. We are currently working toward the realization of two-dimensional phaselocked arrays of anti-guided VCSEL's.

Sandia is a multiprogram laboratory operated by Sandia Corporation for the United States Department of Energy under contract No. DE-AC04-94AL85000. 


\section{References:}

${ }^{1}$ J. P. van der Ziel, D. G. Deppe, N. Chand, G. J. Zydzik, S. N. G. Chu, IEEE J. Quantum Electron. 26, 1873 (1990).

${ }^{2}$ M. Orenstein, E. Kapon, N. G. Stoffel, J. P. Harbison, L. T. Florez, and J. Wullert, Appl. Phys. Lett. 58, 804 (1991).

${ }^{3}$ M. E. Warren, P. L. Gourley, G. R. Hadley, G. A. Vawter, T. M. Brennan, B. E. Hammons, and K. L. Lear, Appl. Phys. Lett. 61, 1484 (1992).

${ }^{4}$ D. Botez, L. Mawst, P. Hayashida, G. Peterson, and T. J. Roth, Appl. Phys. Lett. 53, 464 (1988).

${ }^{5}$ L. J. Mawst, D. Botez, T. J. Roth, and G. Peterson, Appl. Phys. Lett. 55, 10 (1989).

${ }^{6}$ G. R. Hadley, D. Botez, and L. J. Mawst, IEEE J. Quantum Electron. 27, 921 (1991).

${ }^{7}$ G. R. Hadley, Opt. Lett. 20, 1483 (1995).

${ }^{8}$ C. J. Chang-Hasnain, Y. A. Wu, G. S. Li, G. Hasnain, K. D. Choquette, C. Caneau, and L. T. Florez, Appl. Phys. Lett. 63, 1307 (1993).

${ }^{9}$ T.-H. Oh, O. B. Shchekin, and D. G. Deppe, IEEE Photon. Technol. Lett. 10, 1064 (1998).

${ }^{10}$ K. D. Choquette, G. R. Hadley, H. Q. Hou, K. M. Geib, and B. E. Hammons, Electron. Lett. 34, 991 (1998).

${ }^{11}$ K. D. Choquette, R. P. Schneider, K. L. Lear, and K. M. Geib, Electron. Lett. 30, 2043 (1994). 


\section{Figure Captions:}

Fig. 1. Graph (a) schematically shows the layer boundaries above and below the optical cavity. As indicated, a patterned etch decreased the cavity length to yield anti-guided regions. Graph (b) shows the longitudinal $\mathrm{k}$-vector component required to satisfy the local resonance condition. Light emitted within each etched region has a vertical wavevector $\mathbf{k}$ and can leak into the coupling region provided that the wavevector tilts to $\mathbf{k}^{\prime}$.

Fig. 2. Total output power versus total injected current for two anti-guided VCSEL's separated by $8 \mu \mathrm{m}$. The device was heated to $50^{\circ} \mathrm{C}$ and current was injected asymmetrically, with $46 \%$ (54\%) entering the left (right) electrode. The external threshold voltage was $2.7 \mathrm{~V}$. The inset schematically shows the coupled-VCSEL structure. The 3-nm etched VCSEL's are represented by the two gray squares, the penetration of the oxide layers into the mesa is indicated by the hatched region, and the electrodes that contacted the top p-type GaAs layer are shaded black.

Fig. 3. Images of the emission from two coupled VCSEL's separated by $8 \mu \mathrm{m}$ (column a) and 20 $\mu \mathrm{m}$ (column b). Row 1 shows near-field images of the laser output. Row 2 shows far-field images of the phase-locked VCSEL output, obtained by injecting roughly equal currents into the left and right electrodes. Row 3 shows far-field images of the emission from a single VCSEL, obtained by injecting current into only the left electrode.

Fig. 4. Part (a) shows a high-magnification near-field image of two phase-locked VCSEL's separated by $8 \mu \mathrm{m}$. (Two separate images were superimposed: an attenuated image of the laser output and an un-attenuated image of the mesa and electrodes.) For comparison, part (b) shows the result of a two-dimensional numerical simulation of the optical mode. The simulated $2.5-\mathrm{nm}$ layer discontinuities at lateral positions of 6 and $14 \mu \mathrm{m}$ are almost invisible. Presumably, the center electrode in part (a) hides the middle intermediate lobe predicted in part (b). 


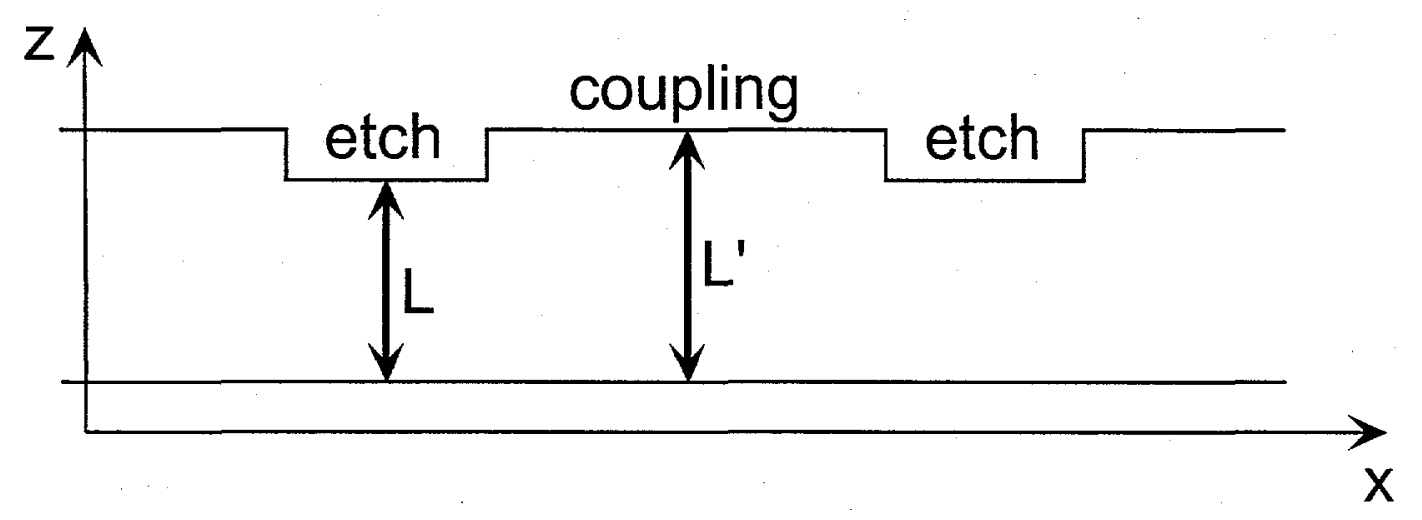

(a)

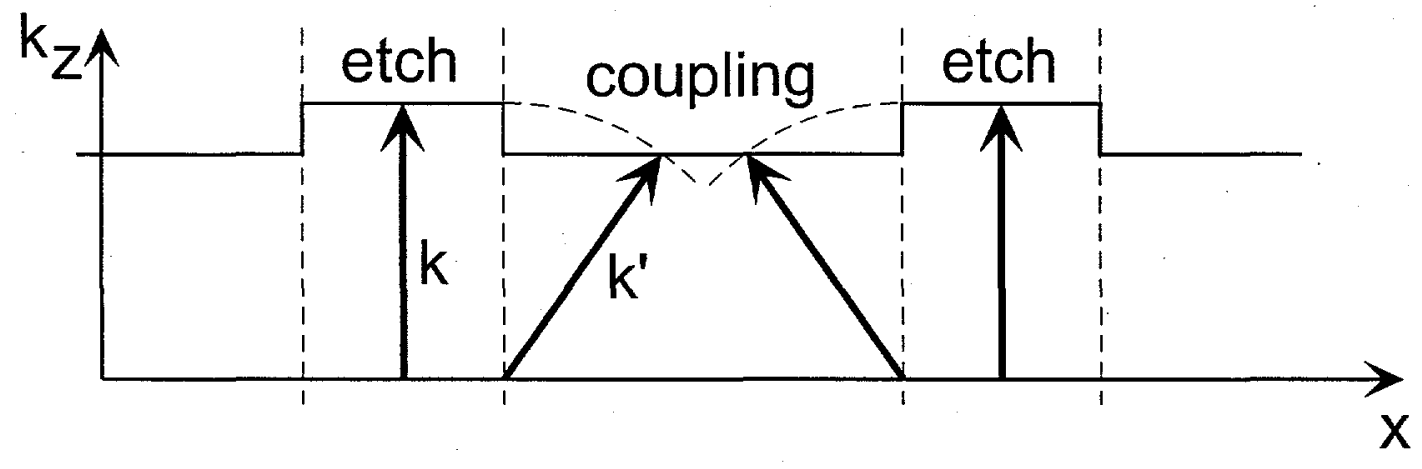

(b)

Fig. 1

D.K. Serkland et al.

9 


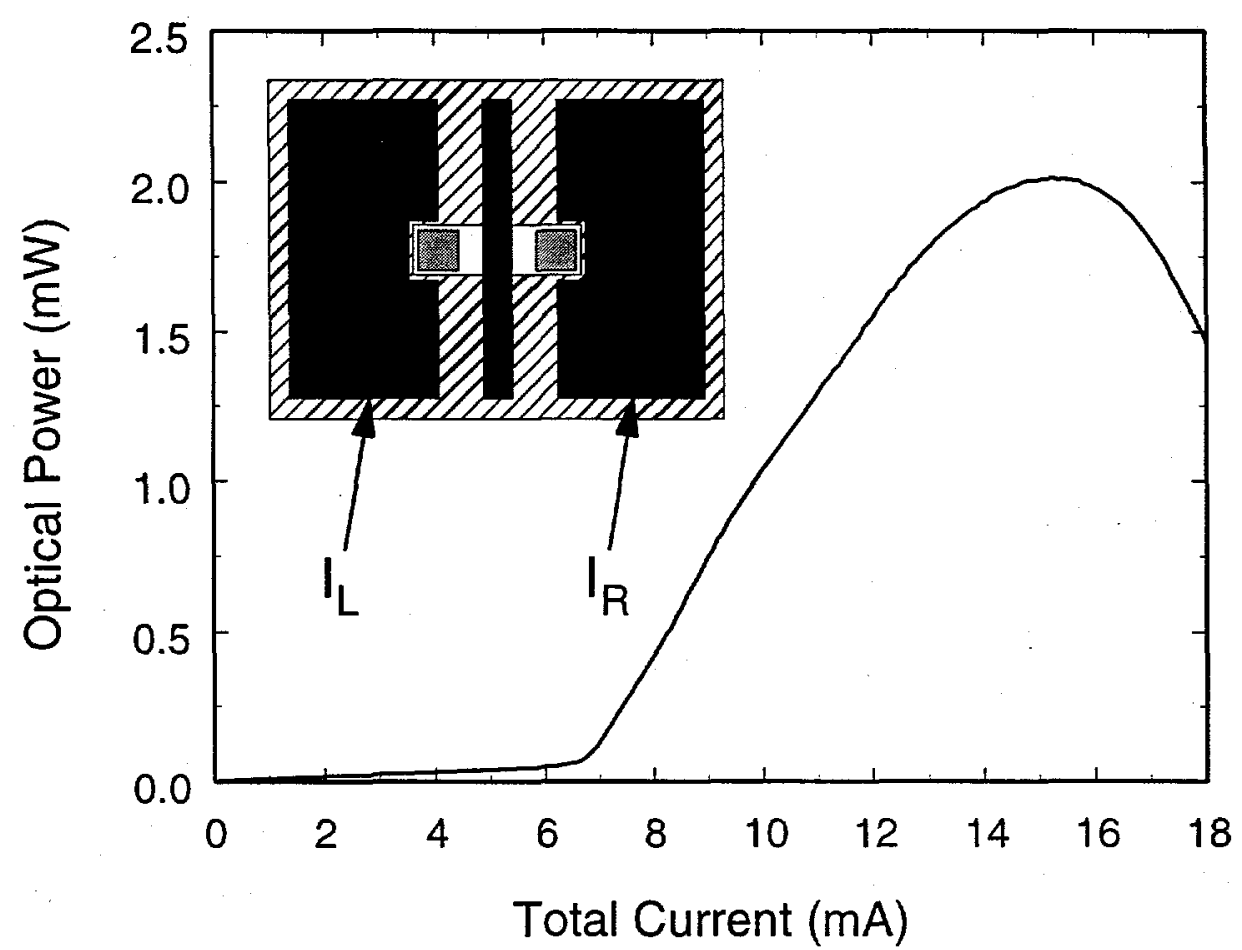

Fig. 2

D.K. Serkland et al.

10 
(a)
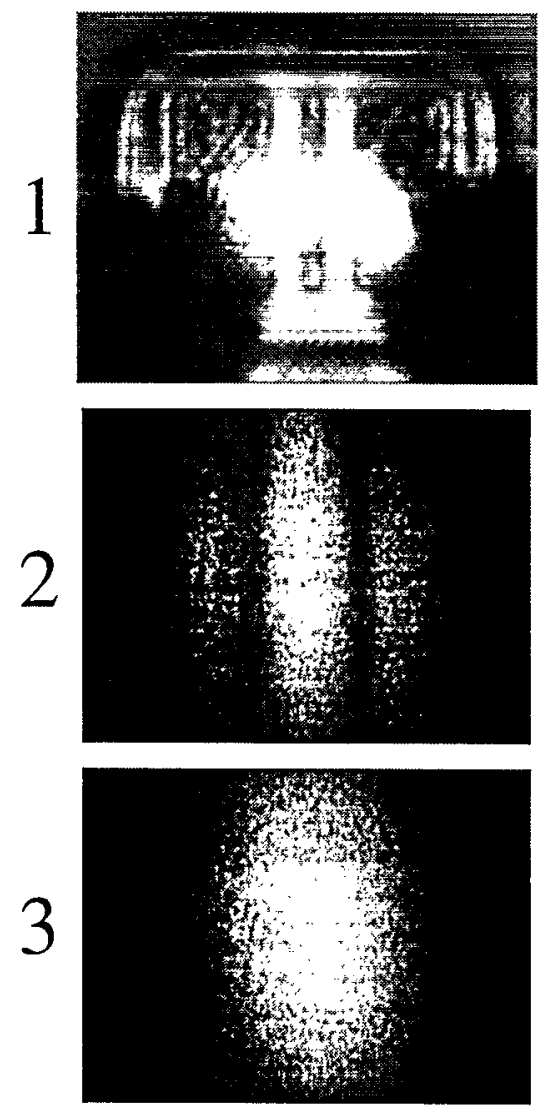

(b)
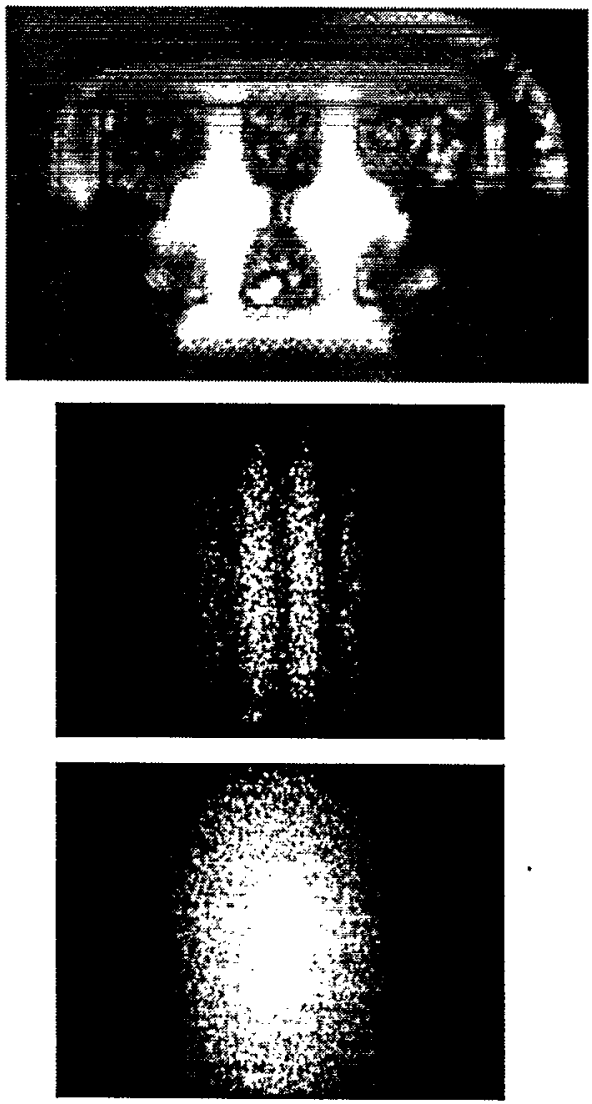

Fig. 3

D.K. Serkland et al. 


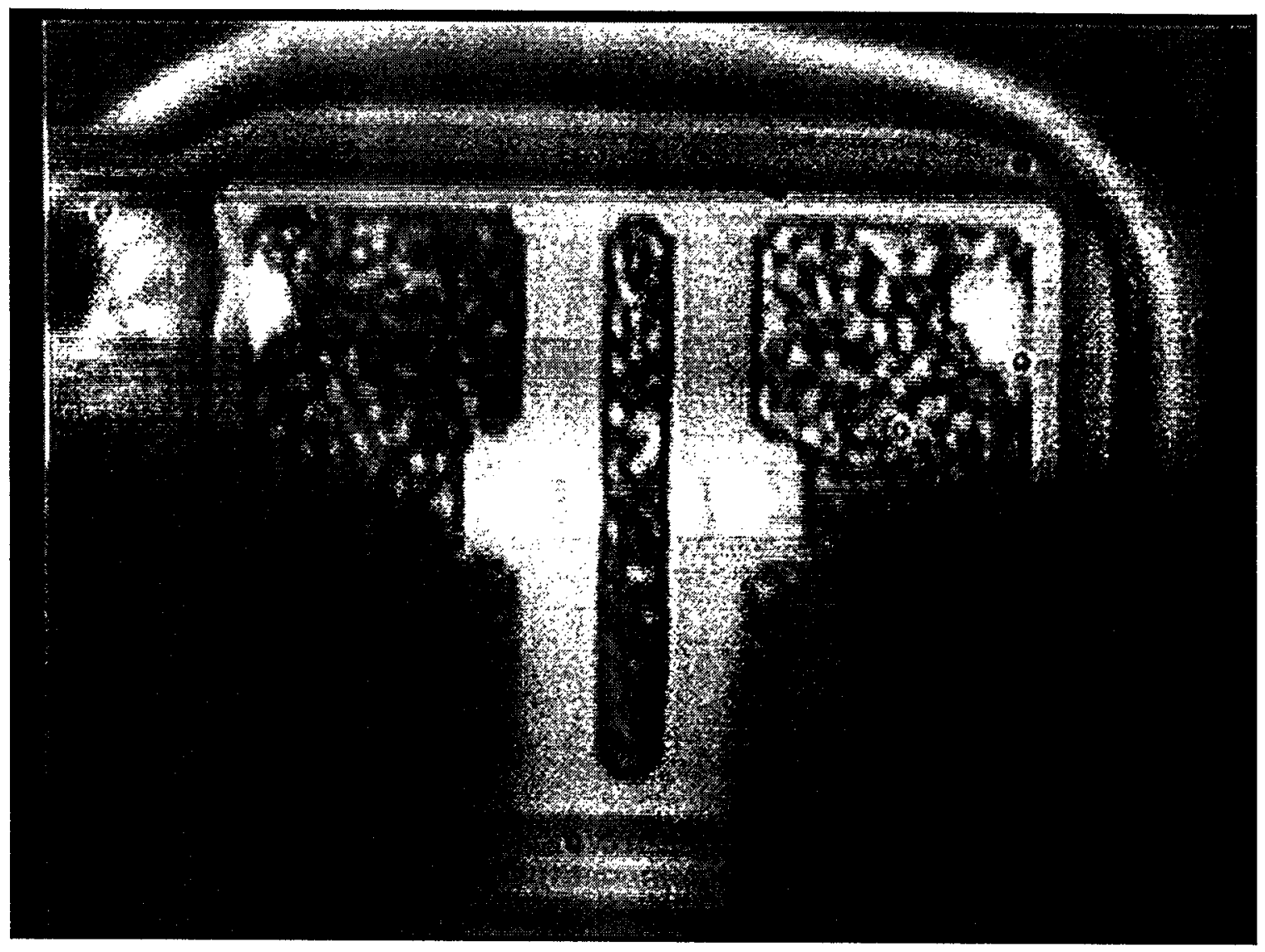

Fig. $4(a)$

D.K. Serkland et al. 


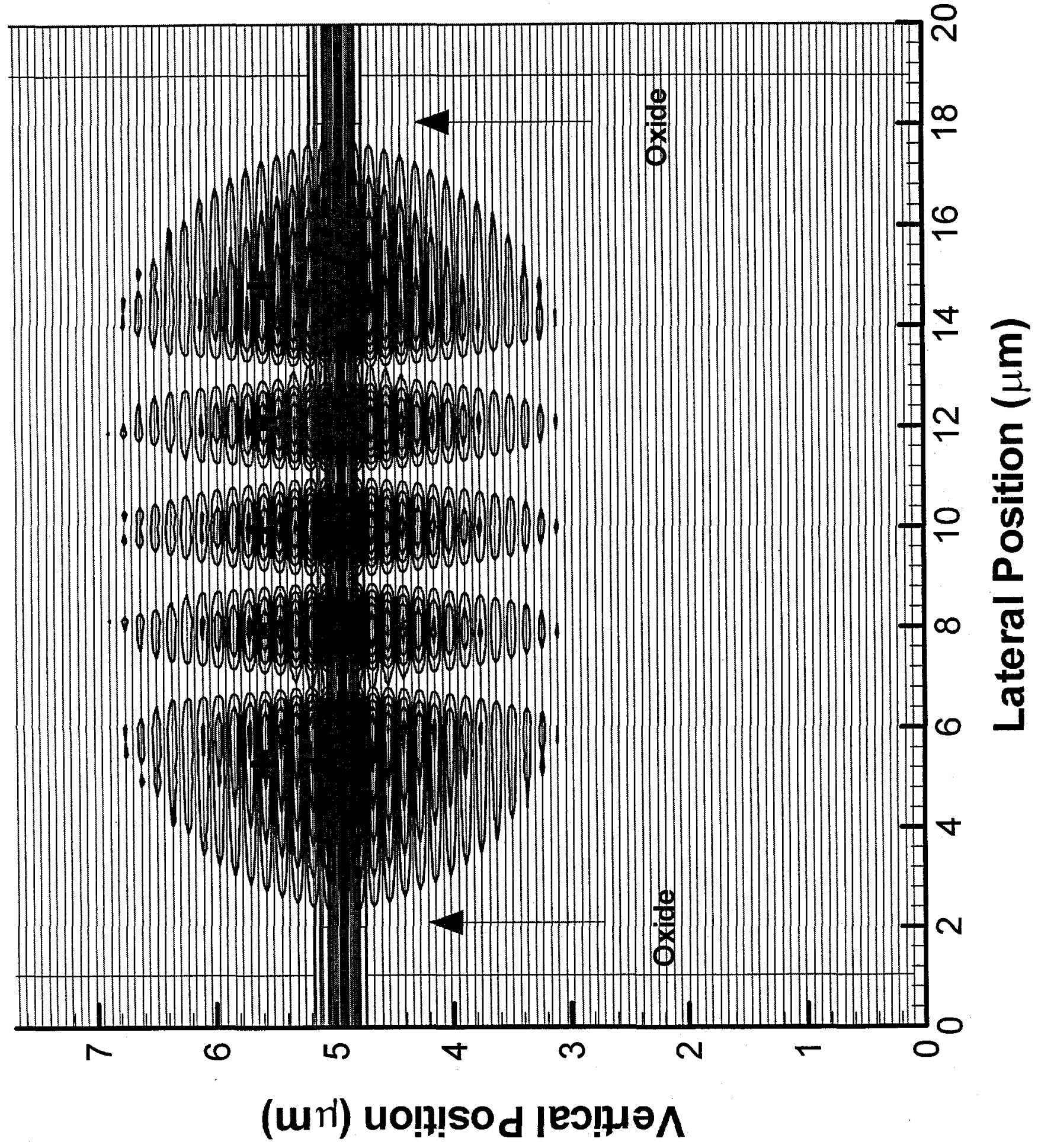

Fig. $4(b)$ D.K.Serkland et al. 